\title{
Vibrio chromosomes share common history
}

\author{
Benjamin C Kirkup Jr*1, LeeAnn Chang1', Sarah Chang², Dirk Gevers³ and Martin F Polz'
}

\begin{abstract}
Background: While most gamma proteobacteria have a single circular chromosome, Vibrionales have two circular chromosomes. Horizontal gene transfer is common among Vibrios, and in light of this genetic mobility, it is an open question to what extent the two chromosomes themselves share a common history since their formation.
\end{abstract}

Results: Single copy genes from each chromosome (142 genes from chromosome I and 42 genes from chromosome II) were identified from 19 sequenced Vibrionales genomes and their phylogenetic comparison suggests consistent phylogenies for each chromosome. Additionally, study of the gene organization and phylogeny of the respective origins of replication confirmed the shared history.

Conclusions: Thus, while elements within the chromosomes may have experienced significant genetic mobility, the backbones share a common history. This allows conclusions based on multilocus sequence analysis (MLSA) for one chromosome to be applied equally to both chromosomes.

\section{Background}

In traditional dogma, bacteria have one chromosome and a number of smaller DNA entities, like plasmids, which are propagated across generations unlinked to the chromosome. However, when bacteria have two chromosomes, are they permanently paired or do these physical entities recombine frequently relative to genes on these chromosomes? Since 1998, it has been known that some gamma proteobacteria have two chromosomes [1-3]. This followed discoveries that various other proteobacteria, namely alpha proteobacteria $[4,5]$ and beta proteobacteria [6], could have multiple chromosomes as well. An initial debate occurred over whether the second Vibrio chromosome was really a 'chromosome' or whether it was merely a 'megaplasmid' [3,7]. The arguments for considering the second replicon a chromosome centered on its considerable size, essential gene content [8] and consistent stoichiometry. We can now add to that a unique replication machinery $[9,10]$ that operates independently but in a coordinated fashion [11] with synchronous termination and thus consistent stoichiometry [12,13]. It is now accepted that most, perhaps all, Vibrionaceae (including the genera Vibrio and Photobacteria) have two chromosomes [14].

* Correspondence: bckirkup@post.harvard.edu

1 Dept. of Civil and Environmental Engineering, 15 Vassar Street, Cambridge MA 02139, USA

Full list of author information is available at the end of the article
Genome analysis of the Vibrios rapidly uncovers variation even among closely related strains. Not only do the genome sizes differ widely [15], but even among conserved genes, there is incongruity among the inferred phylogenies. This is the well-accepted signature of horizontal gene transfer and homologous recombination. Gene organization also differs among sequenced strains, indicating large-scale genetic mobility. Individual genes and entire operons may be mobile among Vibrio [16-20]. In particular, Chromosome II varies widely in size and organization [14,21]. Further, many Vibrio carry (and presumably exchange) plasmids. Though it may seem unusual to expect as large a quantity of DNA to be transferred as an entire chromosome, there is evidence that Vibrio have experienced a transfer on that magnitude even recently: The putative $V$. vulnificus hybridization leading to biotype 3 involves very large quantities of DNA being transferred among $V$. vulnificus strains to create a hybrid strain almost evenly split in contributions from biotypes 1 and 2 [22]. However, the hybridization event involves loci from both chromosomes being transferred and appears to have preserved their associations with those chromosomes. As such, it does not appear to have been an exchange of chromosomal partners, but it raises the possibility that chromosomal exchange may have been an evolutionary mechanism within the Vibrionaceae. 
The function of a second chromosome, and of multichromosomality in general, has been the subject of speculation $[2,14,23]$. That many of the genes on the Vibrio Chromosome II have specific environmental functions has been noted, and the role of the second chromosome in habitat adaptation has been tested experimentally [23]. $\mathrm{Xu}$ et al demonstrated that when V. cholera was grown in an animal host (rabbit ileal loop) a general shift in gene expression favored up-regulation of genes on the second chromosome relative to the gene expression profiles in exponential growth in vitro. This experimental data paired with the gross similarities among the chromosome I from all sequenced Vibrio and the great diversity of chromosome II, suggests that the second chromosome represents a collection of accessory elements and might be mobilized wholesale leading to a complete shift in habitat or niche $[2,14]$.

'Vibrio phylogenies' that are built using MLSA or single-copy conserved genes typically use genes located on chromosome I [15,24-34] with the exception of intra-specific typing schemes for pathogens $[17,22]$. This is a sideeffect of choosing stable, conserved, essential, single copy genes. However, it provides little assurance of representing the history of the entire genome given that Chromosome II is excluded from the analyses.

Given the high degree of mobility Vibrio genetic elements are presumed to have, it is possible that the two chromosomes have distinct and conflicting histories. There are currently 9 completely assembled Vibrio genomes available in the public databases and over 30 partially sequenced genomes. We explored these genomes to construct phylogenies for each of the two chromosomes using three approaches. First, single copy genes from each chromosome were assembled en suite and a phylogeny for each chromosome was inferred from these concatenated sequences. Second, the organization and gene content at the origins of replication of each chromosome (OriI and OriII for chromosomes I and II, respectively) were studied. Third, the genes from near the two chromosomal origins of replication were studied and their phylogenies estimated individually.

\section{Results and Discussion}

\section{Chromosome Phylogenies}

The inferred phylogenies for the two chromosomes are congruent (Figures 1 and 2) and contain the expected major features, such as Photobacterium being basal to the Vibrionaceae and V. fisheri forming the next most basal clade. There are no unexpected sister taxa. The results of this analysis are compatible with published multi-locus analyses. However, instead of using 6 or 8 genes commonly used in MLSA, this analysis included 142 genes from chromosome I and 42 from chromosome II. These single copy genes include a range of functions including metabolism, information processing, flagellar structure and cytoskeletal components; as such, they represent sampling points from various pathways and genomic sections from around the entire genome. The concatenation of these well conserved genes provides a shared signal for the chromosomes as a whole, despite only composing a small fraction of the entire genome. The genes included in the analysis are listed under Additional files 1 and 2. The chromosome I tree is easily rooted by the various other genomes included in the analysis. All of these other clades fell together along accepted taxonomic lines. The most closely related strains in the tree are the $V$. cholerae strains; that clade is effectively unresolved because the internal distances are too short. The chromosome II tree cannot be rooted in the same manner as chromosome I because there is no obviously available outgroup: the chromosome II of $P$. atlantica is not homologous to the chromosome II of the Vibrionaceae being analyzed. However, rooting it identically by using the information from the chromosome I tree preserves the branching order of each tree. Thus, the 'mean field' approximation for the phylogeny of the two chromosomes is congruent at the species level. There is insufficient resolution among $V$. cholerae strains and too few members of other species to make inferences at a finer phylogenetic scale.

\section{Origin of Replication Organization}

The second method of analysis, studying the gene organization at the origins of replication (Ori), supported the finding that the two chromosomes share a single phylogeny at the species level. This method of analysis was more advantageously applied to chromosome II than chromosome I: Gene order in the region immediately surrounding the chromosome I origin appears too highly conserved between species to provide robust data on its phylogeny (Figure 3; expanded in Additional files 3 and 4). However, gene content is informative in that region suggesting that the species largely conform to the expected clustering even though the tree is not well supported (Figure 3). The difficulties are caused by a paucity of organizational changes that differentiate species at OriI - such as the inversion of three genes that sets apart the $V$. fisheri. Frequently, a change is unique to a sequenced strain and not shared by other members of its species. This can be extraordinarily disruptive of a distance estimate if the number of unique differences is large. In particular, at least three obvious saltations in the gene content introduce spikes of noise. In V. cholerae B33, an apparently mobile genetic region has imposed itself very close to the origin of replication. These 18 genes, almost as large as the region to be compared, interrupt an otherwise absolutely conserved region shared by the other Vibrio cholerae. A 9 gene region in Photobacterium sp. SKA34 contains several transposon and transposase 


\section{2}

Oceanobacter sp. RED65

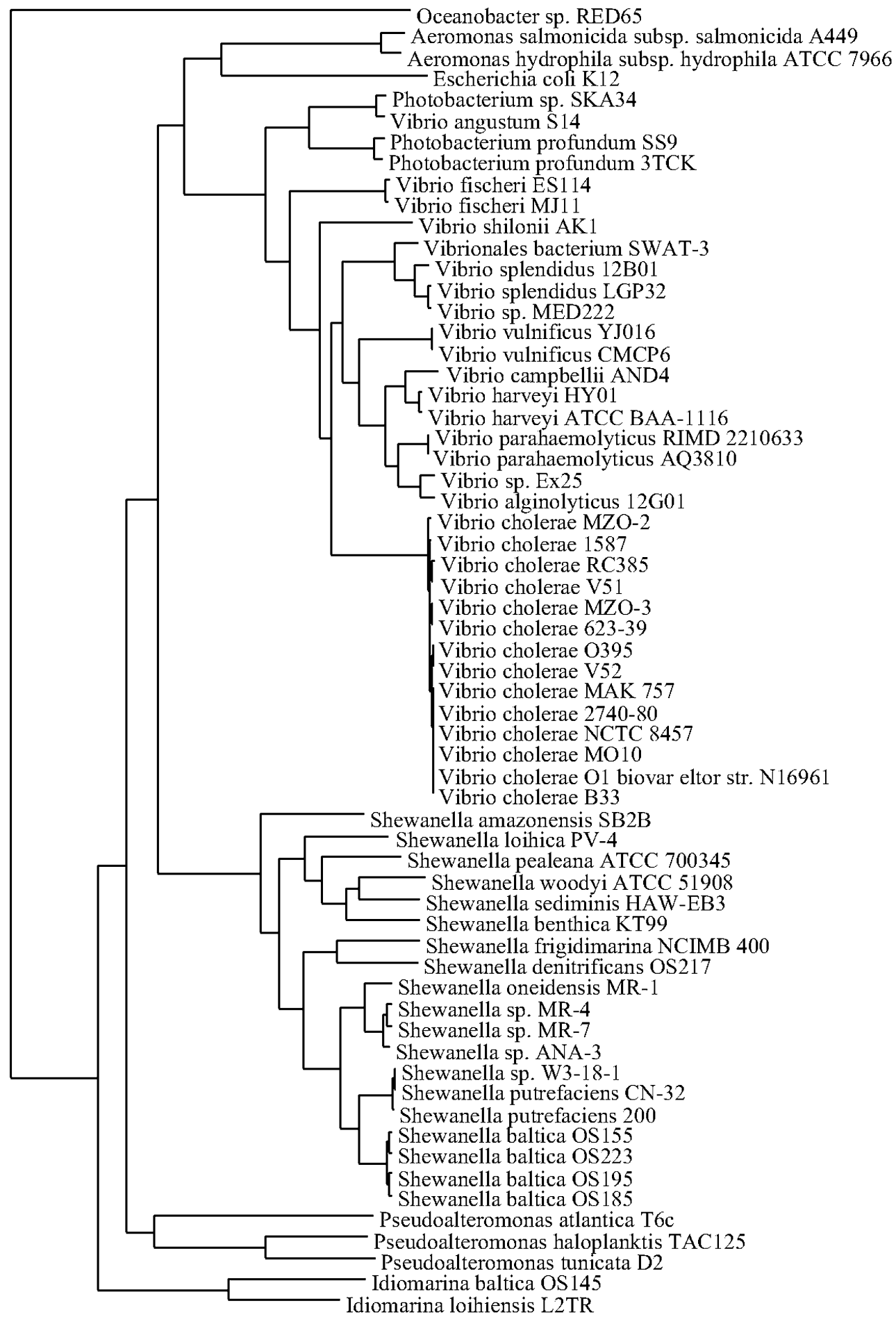

Figure 1 Tree (Chromosome I). Inferred mean-field phylogeny of Chromosome I derived from a sampled concatenated gene sequence of singlecopy orthologs distributed around the entire Chromosome I. The species tree is fully resolved and has 100\% bootstrap support on all nodes outside of $V$. cholerae (1000 replicates). The list of genes and included locus tags is found in Additional file 1, supplementary materials. 


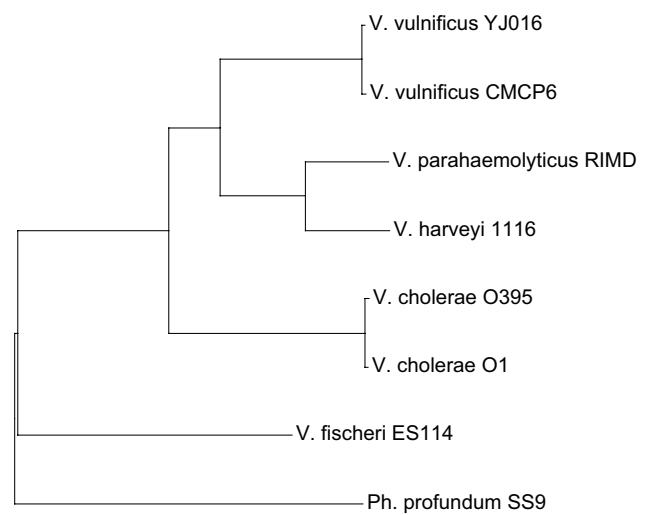

Figure 2 Tree (Chromosome II). Inferred mean-field phylogeny of Chromosome Il derived from a sampled concatenated gene sequence of single-copy orthologs distributed around the entire Chromosome II. The species tree is fully resolved and has $100 \%$ bootstrap support on all nodes (10000 replicates). The list of genes and included locus tags is found in Additional file 2, supplementary materials. Only closed genomes were included in this analysis.

genes. Similarly, 16 gene region in Vibrio splendidus MED222 interrupts an otherwise conserved region with a number of secretory system genes; it lacks apparent mobility elements which would explain its origin. Among the photobacteria, the flanking regions sometimes differ dramatically, as well, which disturbs the phylogeny with a very long branch, and the Vibrio cholerae appear to have inverted the entire region - but this would not impact a gene content analysis.

Gene presence/absence data is more informative in the regions surrounding the origin of chromosome II, even though across the breadth of the Vibrionaceae, the areas adjacent to the origin of replication have been relocated to such an extent that it is not practical to reconstruct their movement from as few genomes as are currently available. This is not unexpected, given how thoroughly shuffled chromosome II is relative to chromosome I [21]; see also Additional file 5 to explore the global rearrangement of chromosome II. Within a relatively short distance of the origin, however, genes can be reliably identified as orthologous and used in a presence/absence analysis. The origin was extended in each direction by 10 $\mathrm{kb}$. As described in the methods, a gene presence/ absence tree was constructed and this led to a distance tree entirely consistent with the mean-field approximation across Chromosomes I and II (i.e. Figures 1 and 2).

\section{Origin of Replication Genes}

The phylogenies estimated for each of the gene families near the origin support the estimations derived from the two chromosomes overall. This third method of analysis led thus to the same conclusion as the other two. Table 1 lists the genes studied at each origin, focusing on their gene phylogeny, while Table 2 specifies the longer annotation names for the genes used in Table 1 and the type of data (DNA or AA) used to create the trees. The genes within the Ori regions are naturally subject to horizontal gene transfer and mutational noise, like all other genes. Two of them are too conserved or too noisy to present a clear phylogenetic signal over the Vibrionales. In these cases, ALrT (approximate likelihood ratio test) and bootstrap support are lacking across the entire tree $(2 / 28$ genes on chromosome I, 0 on chromosome II). Many other trees have limited support for individual clades. Clades with less than 0.05 ALrT [35] support or less than $70 \%$ bootstrap support were reduced to polytomies. In addition, the long branch of $V$. cholerae sometimes distorts other elements in the tree. In $8 / 28$ trees from chromosome I and 2/12 trees derived from chromosome II, removing the cholera clade from the tree also restored a topology consistent with the mean-field tree in the other portions of the tree where previously it had been inconsistent with the hypothesis (labeled B in the first column of the table). Finally, one clade (V. parahaemolyticus, $V$. alginolyticus, $V$. campbellii, $V$. harveyi) was reliably monophyletic but presented numerous permutations in its internal structure. At OriI 9/28 genes presented diverse variants in this clade; at OriII, $3 / 12$ genes presented variability within this clade. Ignoring this variation, 16/28 genes from chromosome I and 10/12 genes from chromosome II confirm the chromosomal phylogenies inferred by the above methods (labeled A). Finally, the remaining two genes on chromosome I lead to inferences that conflict with the others by placing $V$. splendi$d u s$ in the $V$. fischeri clade (basal to its expected position, see Figure 4). Genes in OriI show more variation in phylogeny but fewer genes are available for study in OrilI.

\section{Conclusions}

Rampant horizontal gene transfer and plasmid exchange might create doubt as to the fidelity of paired chromosomes to one another. Further, this genetic mobility can create serious difficulties for anyone reconstructing a phylogeny for something as large as a chromosome, just as they do for someone inferring organismal and species phylogenies. Here, these difficulties have been overcome by using a range of methods that operate at different temporal and genetic scales. At the smallest scale, a number of individual gene phylogenies were reconstructed. At an intermediate scale, the gene content of a conserved region was used to infer a phylogeny. At the largest scale, concatenation of predominantly chromosome specific genes (though they may, in other genomes, be transferred among the chromosomes) provided an estimate of the 

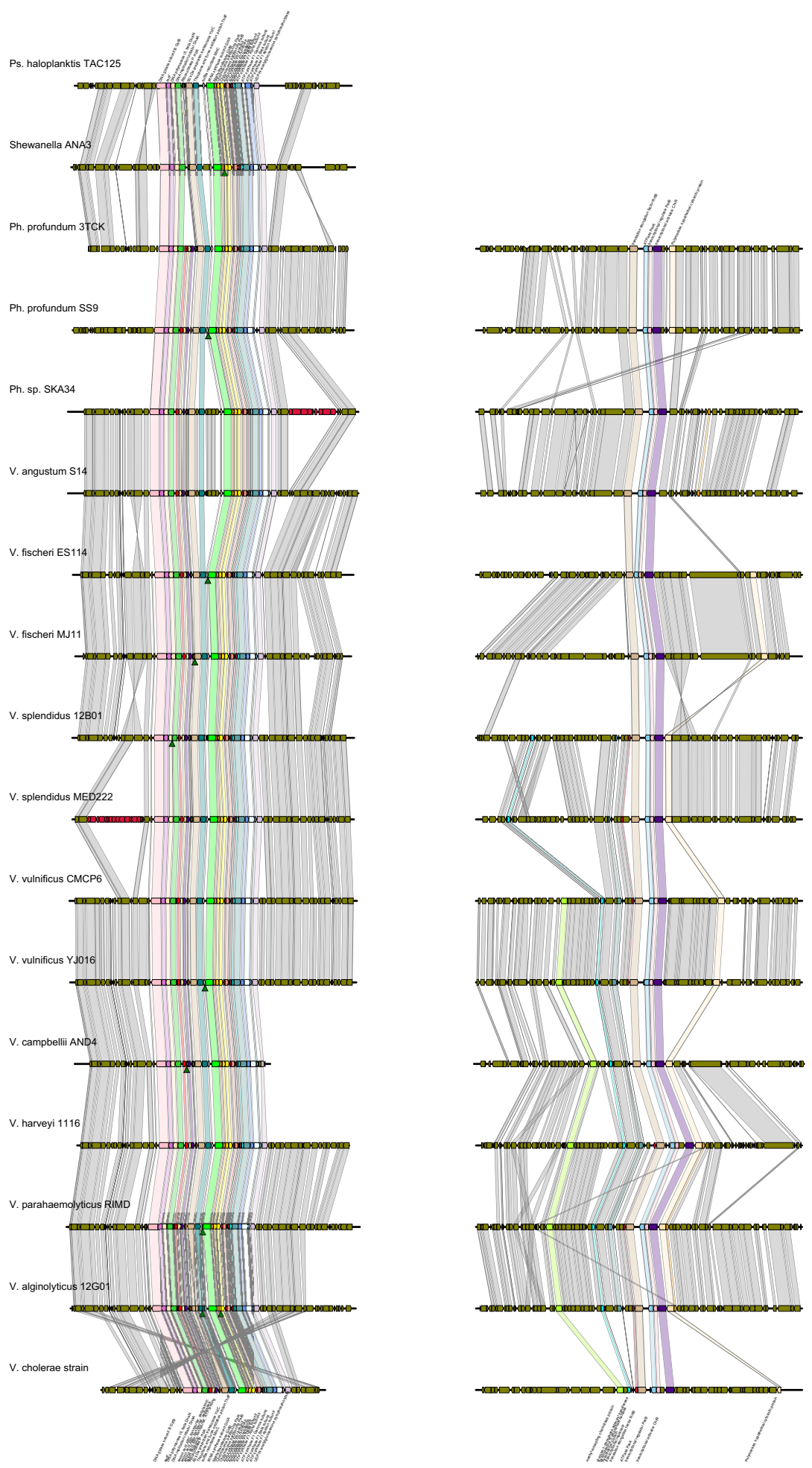

Figure 3 Oril and Orill synteny figures. The two origin regions of (A) Chromosome I and (B) Chromosome II. Open reading frames called in the annotated genomes are polygons pointing in the direction of their orientation. Colors label the open reading frames analyzed individually in estimating the phylogeny of the origin. The expanded figures with all labels are found in Additional files 3 and 4, supplementary materials. 
Table 1: Gene phylogenies for Oril and Orill.

\begin{tabular}{|c|c|c|c|c|}
\hline & $\begin{array}{c}\text { Relation to } \\
\text { hypothesized } \\
\text { chromosome } \\
\text { phylogeny }\end{array}$ & Sequence set & Resolution & $\begin{array}{c}\text { V. alginolyticus/V. } \\
\text { parahaemolyticus } \\
\text { clade }\end{array}$ \\
\hline & $\begin{array}{c}\text { Consistent (A) } \\
\text { Conditionally } \\
\text { consistent (B) } \\
\text { Uninformative (U) } \\
\text { Inconsistent (I) }\end{array}$ & $\begin{array}{c}\text { Complete(C) } \\
\text { Incomplete (P) }\end{array}$ & $\begin{array}{c}\text { Full (F) } \\
\text { Partial (N) } \\
\text { Unresolved (U) }\end{array}$ & $\begin{array}{c}\text { Consistent with } \\
\text { consensus(-) } \\
\text { Scrambled (X) } \\
\text { Other issues (see } \\
\text { text) (O) } \\
\text { Tree uninformative } \\
\text { (n/a) }\end{array}$ \\
\hline \multicolumn{5}{|l|}{ C1 } \\
\hline LysR & A & $P$ & $\mathrm{~N}$ & - \\
\hline MDR & A & $P$ & $\mathrm{~F}$ & - \\
\hline UDP & I & C & $\mathrm{N}$ & - \\
\hline Epsilon & B & C & $\mathrm{N}$ & $\mathrm{O}$ \\
\hline Beta & B & C & $\mathrm{N}$ & $\mathrm{O}$ \\
\hline Gamma & B & $P$ & $\mathrm{~N}$ & - \\
\hline Alpha & A & C & $\mathrm{N}$ & - \\
\hline Delta & A & C & $\mathrm{N}$ & $x$ \\
\hline Bsub & A & C & $\mathrm{N}$ & - \\
\hline Csub & U & C & $U$ & $\mathrm{n} / \mathrm{a}$ \\
\hline Asub & 1 & C & $\mathrm{N}$ & - \\
\hline Isub & A & C & $\mathrm{N}$ & $\mathrm{x}$ \\
\hline ParB & A & C & $\mathrm{N}$ & - \\
\hline ParA & A & C & $\mathrm{N}$ & - \\
\hline GidB & A & C & $\mathrm{N}$ & - \\
\hline GidA & B & C & $\mathrm{N}$ & - \\
\hline MioC & B & C & $F$ & $x$ \\
\hline ThdF & B & C & $\mathrm{N}$ & $x$ \\
\hline
\end{tabular}


Table 1: Gene phylogenies for Oril and Orill. (Continued)

\begin{tabular}{|c|c|c|c|c|}
\hline YidC & $A$ & $C$ & $\mathrm{~N}$ & $x$ \\
\hline RnpA & A & $C$ & $\mathrm{~N}$ & - \\
\hline $\mathrm{RpmH}$ & $U$ & $\mathrm{P}$ & $U$ & $\mathrm{n} / \mathrm{a}$ \\
\hline $\mathrm{ABC} 1$ & $A$ & $P$ & $\mathrm{~N}$ & $x$ \\
\hline $\mathrm{ABC2}$ & A & $P$ & $\mathrm{~N}$ & - \\
\hline $\mathrm{ABC} 3$ & $A$ & $P$ & $\mathrm{~N}$ & - \\
\hline DnaA & A & $C$ & $\mathrm{~N}$ & - \\
\hline DnaN & A & $C$ & $\mathrm{~N}$ & - \\
\hline RecF & B & $C$ & $\mathrm{~F}$ & $x$ \\
\hline GyrB & B & $C$ & $\mathrm{~F}$ & - \\
\hline
\end{tabular}

\section{C2}

\begin{tabular}{|c|c|c|c|c|}
\hline MetC & A & $P$ & $\mathrm{~F}$ & $x$ \\
\hline GluP & B & $P$ & $\mathrm{~N}$ & $x$ \\
\hline PyrD & A & $P$ & $N$ & - \\
\hline GTP & A & $P$ & $\mathrm{~F}$ & - \\
\hline Hyp & A & $P$ & $N$ & - \\
\hline TraR & A & $P$ & $\mathrm{~N}$ & - \\
\hline RctB & A & $C$ & $\mathrm{~N}$ & - \\
\hline ParA2 & A & $C$ & $\mathrm{~N}$ & - \\
\hline ParB2 & A & $C$ & $N$ & - \\
\hline ChrR & A & $C$ & $\mathrm{~N}$ & $x$ \\
\hline Poly & B & $P$ & $\mathrm{~F}$ & - \\
\hline Chlor & A & $P$ & $\mathrm{~F}$ & - \\
\hline
\end{tabular}

All the genes analyzed are listed. The first column represents whether the estimated phylogeny was consistent with the hypothesized chromosome phylogeny ( $\mathrm{A}$ and $\mathrm{B}$, if removing cholera results in a consistent tree), inconsistent with the hypothesized phylogeny, or simply uninformative. The second column indicates whether all strains were represented for this locus and the third whether there were any clades outside the cholera clade at which the tree was a polytomy because of an uninformative or unsupported node. The fourth column includes cases in which $V$. splendidus is out of place in the tree $(O)$ or where the $V$. parahaemolyticus/V. alginolyticus clade is not the same as in the consensus tree $(\mathrm{X})$. 
Table 2: Gene names.

\begin{tabular}{|c|c|c|}
\hline Short name & Long name & Tree type \\
\hline C1 & & \\
\hline LysR & Transcriptional Regulator LysR & $\mathrm{AA}$ \\
\hline Mdr & Multidrug Resistance Protein & AA \\
\hline UPD & $\begin{array}{l}\text { UDP-N-acetylglucosamine } \\
\text { pyrophosphorylase }\end{array}$ & AA \\
\hline Epsilon & ATP synthase F1 epsilon subunit & DNA \\
\hline Beta & ATP synthase F1 Beta subunit & AA \\
\hline Gamma & ATP synthase F1 Gamma subunit & AA \\
\hline Alpha & ATP synthase F1 Alpha subunit & DNA \\
\hline Delta & ATP synthase F1 Delta subunit & DNA \\
\hline Bsub & ATP synthase F0 B subunit & DNA \\
\hline Csub & ATP synthase F0 C subunit & $\mathrm{n} / \mathrm{a}$ \\
\hline Asub & ATP synthase F0 A subunit & DNA \\
\hline Isub & ATP synthase FO I subunit & DNA \\
\hline ParB & Chromosome Partitioning Protein ParB & AA \\
\hline ParA & Chromosome Partitioning Protein ParA & AA \\
\hline GidB & Glucose inhibited division protein B & AA \\
\hline GidA & Glucose inhibited division protein A & AA \\
\hline MioC & Flavodoxin & AA \\
\hline ThdF & Thiophene and furan oxidation protein & DNA \\
\hline YidC & $60 \mathrm{kDa}$ inner membrane insertion protein & AA \\
\hline RnpA & Ribonuclease P & $\mathrm{AA}$ \\
\hline $\mathrm{RpmH}$ & Ribosomal protein L34 & $\mathrm{n} / \mathrm{a}$ \\
\hline
\end{tabular}


Table 2: Gene names. (Continued)

\begin{tabular}{|c|c|c|}
\hline $\mathrm{ABC1}$ & $\begin{array}{l}\text { Amino acid ABC transporter, ATP-binding } \\
\text { protein }\end{array}$ & AA \\
\hline $\mathrm{ABC2}$ & $\begin{array}{l}\text { Amino acid } A B C \text { transporter, permease } \\
\text { protein }\end{array}$ & $A A$ \\
\hline $\mathrm{ABC} 3$ & $\begin{array}{l}\text { Amino acid } A B C \text { transporter, periplasmic } \\
\text { amino acid-binding portion }\end{array}$ & AA \\
\hline DnaA & Chromosomal DNA replication initiator & AA \\
\hline DnaN & DNA Polymerase III, beta chain & $\mathrm{AA}$ \\
\hline RecF & Recombination Protein $\mathrm{F}$ & DNA \\
\hline GyrB & DNA Gyrase & DNA \\
\hline \multicolumn{3}{|l|}{ C2 } \\
\hline MetC & methyl-accepting chemotaxis protein & $A A$ \\
\hline GluP & glucose-1-phosphate adenylyl transferase & $A A$ \\
\hline PyrD & pyridoxamine 5'-phosphate oxidase & DNA \\
\hline GTP & GTP cyclohydrolase II & DNA \\
\hline Hyp & Hypothetical Protein & AA \\
\hline TraR & transcriptional repressor & DNA \\
\hline RctB & Putative Translation Elongation Factor & $A A$ \\
\hline ParA2 & $\begin{array}{l}\text { ATPases involved in Chromosome } \\
\text { Partitioning }\end{array}$ & $A A$ \\
\hline ParB2 & Predicted Transcriptional Regulator ParB & AA \\
\hline ChrR & Transcriptional Activator ChrR & AA \\
\hline Poly & Polymerase, hypothetical cytosolic protein & AA \\
\hline Chlor & Chloramphenicol Acetyltransferase & $\mathrm{AA}$ \\
\hline
\end{tabular}

The genes surrounding the origins of replication otherwise identified by short names are described by their longer annotation names. In addition, the data (DNA or AA) used to create the trees is listed. This relates to the degree of conservation in the data; more conserved sequences require DNA trees to provide signal, less conserved sequences require AA trees to avoid excessive noise. 
history of the whole chromosome. In each case, the observed patterns were consistent - though, while many individual genes do not present a conflicting individual history, they may not support the hypothesis for lack of signal.

This congruence between the whole of the chromosome and the origin of replication suggests that the region around the origin of replication is either too large to relocate or is difficult to transfer because of its specific function. Individual genes in this region may experience horizontal gene transfer - witness the inclusion of a mobile genetic region in $V$. cholerae B33. Individual genes also appear amenable to transfer, deletion and insertion.

More than being able to create a relative history for each chromosome, it appears that since the origin of the two chromosomes in the ancestral Vibrio, they have continued as a pair. This suggests that they have also followed the cell itself; that we have a consistent phylogeny for the Vibrio species themselves - at least, the ones included in this analysis. Further genome sequencing would allow a similar analysis to provide the 'definitive' phylogeny of the Vibrio, but at much greater effort per strain than for MLSA [33]. MLSA schemes currently devised provide a mean field estimate of the phylogeny of Chromosome I; thus, as they are expanded to include increasing numbers of genes, those phylogenies are expected to agree with the phylogenies derived from studying the origins of replication. This suggests several genes that might be used in an MLSA of the Vibrionaceae, including Alpha, DnaN, and YidC from Chromosome 1 and ParA2 and GluP from Chromosome 2. These genes have potential primer sequences that are hypothetically capable of creating phylogenetic trees with the highest resolution and consistent signal so that they are comparable to the trees found in this study. It is a pleasing conclusion that separate MLSA schemes will not have to be executed for each chromosome independently.

\section{Methods}

\section{Chromosome Phylogenies}

Mean field approximation refers to the generalized phylogeny of the entire chromosome, regardless of differing histories. This was accomplished conceptually by means of concatenated gene trees for single copy homologous genes whose relatives are most easily determined and whose chromosomal affiliation is most certain. The restriction that the genes had to be single copy is meant to limit the analysis to orthologs while excluding paralogs. To select the genes for this analysis, a database of genomes was created. All the available Vibrionaceae (Vibrio and Photobacterium) genomes as well as an assortment of other gamma proteobacterial genomes (Additional file 6) were selected for analysis. All 62 genomes were broken down into lists of ORFs, which were entered into a MySQL database with their DNA and protein sequences as well as other identifying data. The entire suite of protein sequences were BLASTed against each other and the resulting hits were processed with orthoMCL $v 1.4$ to identify protein families [36]. A significant parameter used in orthoMCL was an inflation value of 1.5. Genes representing single copy gene families on the different chromosomes were aligned [37], stripped of their gaps, concatenated, and $100 \mathrm{~kb}$, chosen as individual random sites, was chosen as the input for PhyML [38].

Phylogenies for Vibrio and Photobacterium chromosome I and II were based on the complete and incomplete published genomes with $P$. atlantica and Shewanella $s p$. ANA3 serving as the outgroup. Initially, Pseudoalteromonas haloplanktis was proposed as an outgroup for the chromosome II phylogeny. P. haloplanktis, unlike other sequenced pseudoalteromonads, has a second chromosome. However, that chromosome appears to have a distinct, plasmid-like origin of replication and a GC-skew that indicates unidirectional replication [39]. It shares several genes with the Vibrio chromosome II, but it is unclear how, if at all, it might share a substantive phylogeny with the Vibrio chromosome II. It contains presumably essential housekeeping genes, despite its otherwise plasmid-like features and likely represents a second origin of multi-chromosomality within the gamma proteobacteria. As a result, though genes from $P$. haloplanktis chromosome I were used as an outgroup to Vibrionaceae chromosome I, genes from P. haloplanktis chromosome II were not included in any analysis of Vibrionaceae chromosome II.

Initially, only completed Vibrionaceae genomes were analyzed for phylogeny of chromosome II. The incomplete genomes were then added to the analysis; genes represented multiple times in these genomes were excluded from the analysis. Incomplete genomes of Vibrio cholerae B33, Vibrio harveyi HY01, Vibrio cholera MZO-2, and Vibrio angustum S14 were excluded from this tree because they appeared to be missing members of gene families shared by the other genomes, even quite closely related conspecific strains. Finally, all the selected genes were processed as above, under the assumption that in the incompletely sequenced strains, genes particular to chromosome II in the complete genomes remained on chromosome II. With significantly fewer taxa in chromosome II than chromosome I, comparison for phylogenetic congruence involved eliminating a given taxa from the comparison if it was missing from one of the trees, and only using taxa present in both trees.

\section{Origin of Replication Organization}

The origins of replication were studied first in the complete genomes, where they are identifiable by GC skew, annotation, and common gene content and organization. 


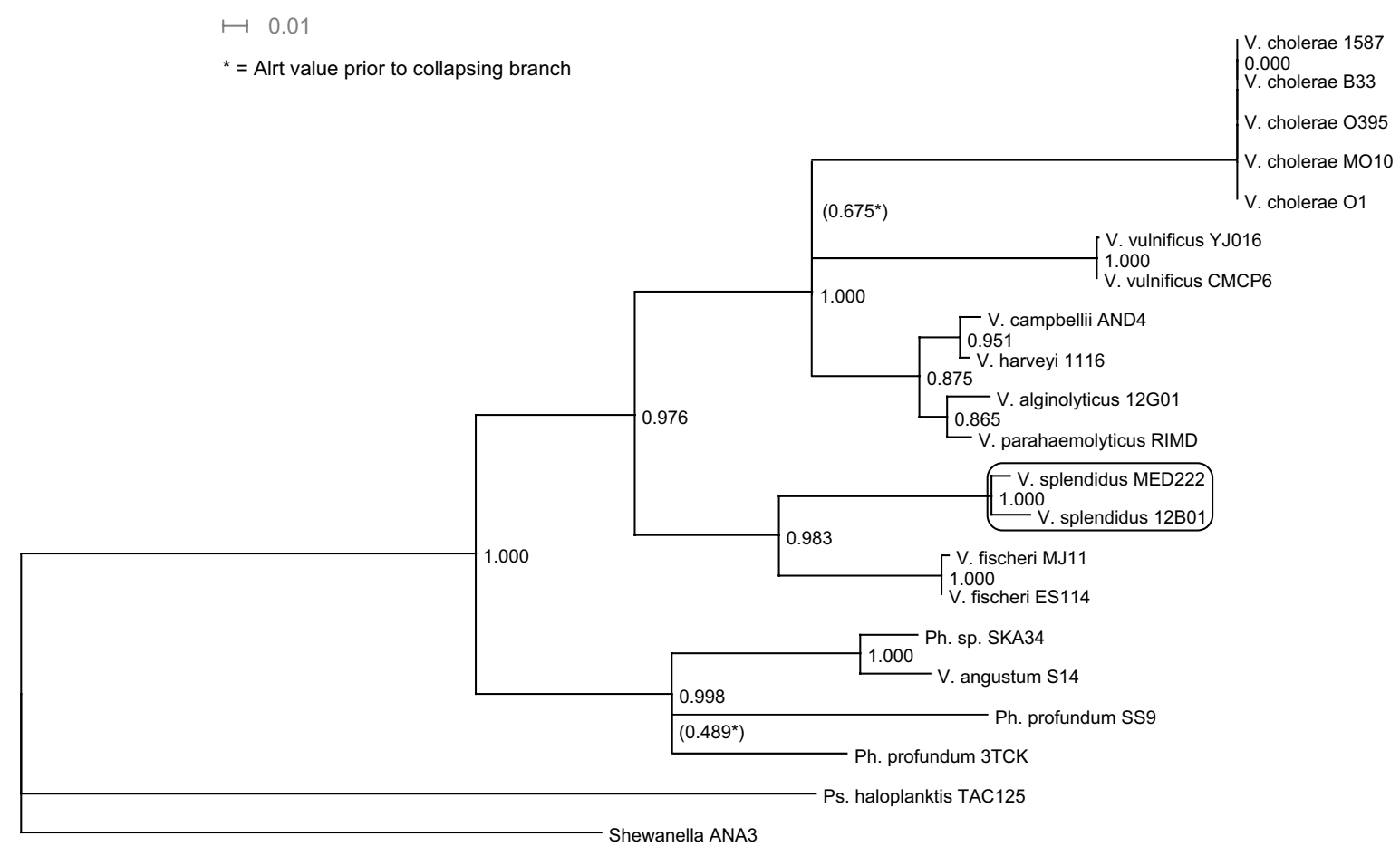

Figure 4 Aberrant tree. Tree inferred from the gene Asub on Chromosome I that is inconsistent with the trees inferred by other methods as described in this paper, including the trees for the individual gene phylogenies at other nearby genes. In this tree, the $V$. splendidus clade is found next to the $V$. fisheri clade, making it basal to its expected position. This tree is also referred to as "l" in Table 1, column 1. As shown, the tree is not fully resolved and branches with low support have been collapsed.

In the incomplete genomes, orthologous regions were identified by both gene content and skew. When the expected gene families and gene order coincided with appropriate shifts in skew, the origin was identified. For unfinished genomes, the origin could not be used in this analysis if it was broken up over several small contigs, but when the entire region was readily assembled in an unmistakable fashion, those contigs were included in the analysis.

The gene families derived from the above database were used to identify orthologs. Four core genes present in virtually all the genomes immediately at the origin were identified and used to anchor the analysis. From their furthest start and stop codons, regions $10 \mathrm{~kb}$ (OrilI) and $20 \mathrm{~kb}$ (OriI) stretching outward were defined. These distances were chosen to balance issues of signal and noise. Particularly for OriI, a shorter region was uninformative because there were too few differences in gene content. For both of the chromosomes, as the regions grew larger, genome rearrangements were encountered that would wash out any signal from similarities in gene content at the origins themselves.
The genes within the selected regions were labeled by family and this data was used to produce a list of genes present in each region. This data was used to produce a pairwise distance estimate.

$$
\text { Dist }=(A-B) / A
$$

Where:

$A=$ the smaller number of labeled genes in either of the two regions (i.e. in genome 1 or 2)

$\mathrm{B}=$ the number of families shared by the two regions (i.e. in the 10 or $20 \mathrm{~kb}$ regions on both genomes)

These pairwise distances were used to construct a square matrix; neighbor.exe from PHYLIP [40] was used to construct a neighbor-joining tree (settings; 10000 jumbles, root, otherwise default).

\section{Origin of Replication Genes}

The genes surrounding the origins of replication were grouped into families by similarity and synteny as detailed above. The phylogenies of the genes were estimated using PhyML-aLRT (settings; AA or DNA depending on data set, otherwise default) and strict consensus trees were created from the phylogenies. The individual 
gene trees were annotated with the necessary rearrangements to fit a largely resolved consensus tree. PhyMLaLRT was employed due to its ability to rapidly calculate the likelihood gain of all branches, allowing those without sufficient signal to be collapsed. As such, the cholera clade in particular contains insufficient divergence to be accurately resolved based on these genes.

The consensus tree arrived at by consensing the individual gene phylogenies estimated from genes near the origins of replication was compared to the trees derived from the other two methods. Common tools used for sequence and tree visualization included Dendroscope [41], BioEdit [42], and Artemis [43].

\section{Additional material}

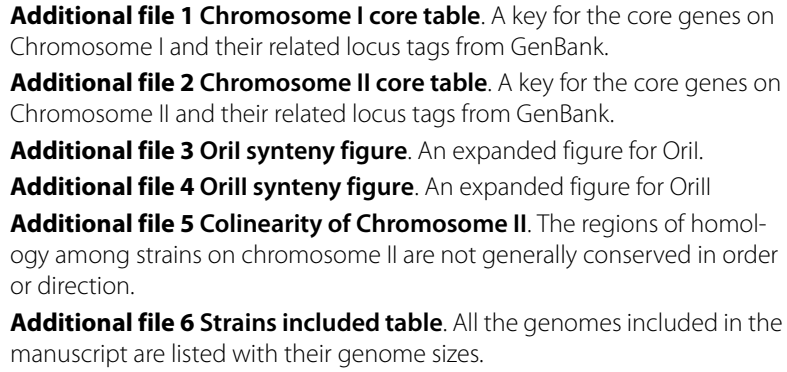

Additional file 6 Strains included table. All the genomes included in the manuscript are listed with their genome sizes.

\section{Authors' contributions}

BCK conceived of the project, generated the methods and drafted the manuscript. LC performed the final version of the analysis for each section and participated in writing the manuscript. SC performed an initial version of the first two analyses. DG developed the database for the research and reviewed drafts of the manuscript. MFP contributed ongoing critical review of the research aims and methods, extensively reviewed and edited the manuscript. All authors have read and approved the final manuscript.

\section{Acknowledgements}

Funding was provided by The Woods Hole Center for Oceans and Human Health (NSF\&NIEHS), the Moore Foundation and DOE-Genomes to Life; computational support was provided by the Darwin Cluster at MIT.

\section{Author Details}

'Dept. of Civil and Environmental Engineering, 15 Vassar Street, Cambridge MA 02139, USA, 2Infectious Diseases, Childrens' Hospital Boston, 200 Longwood Ave., Boston MA 02115, USA and ${ }^{3}$ Microbial Sequencing Center, The Broad Institute of MIT and Harvard, 7 Cambridge Center, Cambridge MA 02142, USA

Received: 27 August 2009 Accepted: 10 May 2010

Published: 10 May 2010

\section{References}

1. Yamaichi Y, lida T, Park K-S, Yamamoto K, Honda T: Physical and genetic map of the genome of Vibrio parahaemolyticus: presence of two chromosomes in Vibrio species. Molecular Microbiology 2002, 31:1513-1521.

2. Heidelberg JF, Eisen JA, Nelson WC, Clayton RA, Gwinn ML, Dodson RJ, Haft DH, Hickey EK, Peterson JD, Umayam L, Gill SR, Nelson KE, Read TD, Tettelin H, Richardson H, Ermolaeva MD, Vamathevan J, Bass S, Qin H, Dragoi I, Sellers P, McDonald L, Utterback T, Fleishmann RD, Nierman WC, White O, Salzberg SL, Smith HO, Colwell RR, Mekalanos JJ, Venter JC, Fraser CM: DNA sequence of both chromosomes of the cholera pathogen Vibrio cholerae. Nature 2000, 406:477-483.
3. Trucksis M, Michalski J, Deng YK, Kaper JB: The Vibrio cholerae genome contains two unique circular chromosomes. Proceedings of the National Academy of Sciences USA 1998, 95:14464-14469.

4. Suwanto A, Kaplan S: Physical and genetic mapping of the Rhodobacter sphaeroides 2.4.1 genome: presence of two unique circular chromosomes. Journal of Bacteriology 1989, 171:5850-5859.

5. Choudhary M, Fu Y-X, Mackenzie C, Kaplan S: DNA Sequence Duplication in Rhodobacter sphaeroides 2.4.1: Evidence of an Ancient Partnership between Chromosomes I and II. Journal of Bacteriology 2004, 186:2019-2027

6. Cheng H-P, Lessie TG: Multiple replicons constituting the genome of Pseudomonas cepacia 17616. Journal of Bacteriology 1994, 176:4034-4042.

7. Kolstø A-B: Dynamic bacterial genome organization. Molecular Microbiology 1997, 24:241-248.

8. Yamaichi Y, Fogel MA, Waldor MK: par genes and the pathology of chromosome loss in Vibrio cholerae. Proceedings of the National Academy of Sciences USA 2007, 104:630-635.

9. Duigou S, Knudsen KG, Skovgaard O, Egan ES, Løbner-Olesen A, Waldor MK: Independent control of replication initiation of the two Vibrio cholerae chromosomes by DnaA and RctB. Journal of Bacteriology 2006, 188:6419-6424.

10. Fogel MA, Waldor MK: A dynamic, mitotic-like mechanism for bacterial chromosome segregation. Genes \& Development 2006, 20:3269-3282.

11. Rasmussen $T$, Jensen RB, Skovgaard O: The two chromosomes of Vibrio cholerae are initiated at different time points in the cell cycle. The EMBO Journal 2007, 26:3124-3131.

12. Egan ES, Løbner-Olesen A, Waldor MK: Synchronous replication initiation of the two Vibrio cholerae chromosomes. Current Biology 2004, 14:R501-R502

13. Srivastava $P$, Fekete RA, Chattoraj DK: Segregation of the replication terminus of the two Vibrio cholerae chromosomes. Journal of Bacteriology 2006, 188:1060-1070.

14. Okada K, lida T, Kita-Tsukamoto K, Honda T: Vibrios commonly possess two chromosomes. Journal of Bacteriology 2005, 187:752-757.

15. Thompson JR, Pacocha S, Pharino C, Klepac-Ceraj V, Hunt DE, Benoit J, Sarma-Rupavtarm R, Distel DL, Polz MF: Genotypic Diversity Within a Natural Coastal Bacterioplankton Population. Science 2005, 307:1311-1313

16. Bisharat N, Amaro C, Fouz B, Llorens A, Cohen DI: Serological and molecular characteristics of Vibrio vulnificus biotype 3: evidence for high clonality. Microbiology 2007, 153:847-856.

17. Bisharat N, Cohena DI, Maidenb MC, Crookd DW, Petoe T, Harding RM: The evolution of genetic structure in the marine pathogen, Vibrio vulnificus. Infection, Genetics and Evolution 2007, 7:685-693.

18. Kasai S, Okada K, Hoshino A, lida T, Honda T: Lateral transfer of the lux gene cluster. Journal of Biochemistry 2007, 141:231-237.

19. Urbanczyk H, Ast JC, Kaeding AJ, Oliver JD, Dunlap PV: Phylogenetic analysis of the incidence of lux gene horizontal transfer in Vibrionaceae. Journal of Bacteriology 2008, 190:3494-3504.

20. Hunt DE, David LA, Gevers D, Preheim SP, Alm EJ, Polz MF: Resource Partitioning and Sympatric Differentiation Among Closely Related Bacterioplankton. Science 2008, 320:1081-1085

21. Reen F, Almagro-Moreno S, Ussery D, Boyd E: The genomic code: inferring Vibrionaceae niche specialization. Nature Reviews: Microbiology 2006, 4:697-704.

22. Bisharat N, Cohen DI, Harding RM, Falush D, Crook DW, Peto T, Maiden MC: Hybrid Vibrio vulnificus. Emerging Infectious Diseases 2005, 11:30-35.

23. Xu Q, Dziejman M, Mekalanos JJ: Determination of the transcriptome of Vibrio cholerae during intraintestinal growth and midexponential phase in vitro. Proceedings of the National Academy of Sciences USA 2003, 100:1286-1291.

24. Dorsch M, Lane D, Stackebrandt E: Towards a phylogeny of the genus Vibrio based on 16S rRNA sequences. International Journal of Systematic Bacteriology 1992, 42:58-63.

25. González-Escalona N, Martinez-Urtaza J, Romero J, Espejo RT, Jaykus L-A DePaola A: Determination of molecular phylogenetics of Vibrio parahaemolyticus strains by multilocus sequence typing. Journal of Bacteriology 2008, 190:2831-2840.

26. González-Escalona N, Whitney B, Jaykus L-A, DePaola A: Comparison of direct genome restriction enzyme analysis and pulsed-field gel electrophoresis for typing of Vibrio vulnificus and their correspondence 
with multilocus sequence typing data. Applied and Environmental Microbiology 2007, 73:7494-7500.

27. Jolley KA, Chan M-S, Maiden MC: mlstdbNet - distributed multi-locus sequence typing (MLST) databases. BMC Bioinformatics 2004, 5:86.

28. Nearhos SP, Fuerst JA: Reanalysis of 5 S rRNA sequence data for the Vibrionaceae with the clustan program suite. Current Microbiology 1987, 15:329-335

29. Nishiguchi MK, Nair VS: Evolution of symbiosis in the Vibrionaceae: a combined approach using molecules and physiology. International Journal of Systematic and Evolutionary Microbiology 2003, 53:2019-2026.

30. Sawabe T, Kita Tsukamoto K, Thompson FL: Inferring the evolutionary history of vibrios by means of multilocus sequence analysis. Journal of Bacteriology 2007, 189:7932-7936.

31. Singh DV, Mohapatra H: Application of DNA-based methods in typing Vibrio cholerae strains. Future Microbiology 2008, 3:87-96.

32. Stine OC, Sozhamannan S, Gou Q, Zheng S Jr, JGM , Johnson JA: Phylogeny of Vibrio cholerae based on recA sequence. Infection and Immunity 2000, 68:7180-7185.

33. Thompson FL, Gevers D, Thompson CC, Dawyndt P, Naser SM, Hoste B, Munn CB, Swings J: Phylogeny and Molecular Identification of Vibrios on the Basis of Multilocus Sequence Analysis. Applied and Environmental Microbiology 2005, 71:5107-5115.

34. Thompson FL, lida T, Swings J: Biodiversity of Vibrios. Microbiology and Molecular Biology Reviews 2004, 68:403-431.

35. Anisimova M, Gascuel O: Approximate likelihood ratio test for branches: A fast, accurate and powerful alternative. Systematic Biology 2006, 55:539-552.

36. Li L Jr, CJS, Roos DS: OrthoMCL: Identification of Ortholog Groups for Eukaryotic Genomes. Genome Research 2003, 13:2178-2189.

37. Thompson JD, Higgins DG, GT J: CLUSTAL W: Improving the sensitivity of progressive multiple sequence alignment through sequence weighting, position-specific gap penalties and weight matrix choice. Nucleic Acids Research 1994, 22:4673-4680.

38. Guindon S, Gascuel O: A simple, fast, and accurate algorithm to estimate large phylogenies by maximum likelihood. Systematic Biology 2003, 52:696-704

39. Médigue C, Krin E, Pascal G, Barbe V, Bernsel A, Bertin PN, Cheung F, Cruveiller S, D'Amico S, Duilio A, Fang G, Feller G, Ho C, Mangenot S, Marino G, Nilsson J, Parrilli E, Rocha EP, Rouy Z, Sekowska A, Tutino ML, Vallenet D, von Heijne G, Danchin A: Coping with cold: The genome of the versatile marine Antarctica bacterium Pseudoalteromonas haloplanktis TAC125. Genome Research 2005, 15:1325-1335.

40. Felsenstein J: PHYLIP (Phylogeny Inference Package). 3.6th edition. Seattle: Department of Genome Sciences, University of Washington; 2005.

41. Huson DH, Richter DC, Rausch C, Dezulian T, Franz M, Rupp R: Dendroscope: An interactive viewer for large phylogenetic trees. $B M C$ Bioinformatics 2007, 8:460.

42. Hall TA: BioEdit: a user-friendly biological sequence alignment editor and analysis program for Windows 95/98/NT. Nucleic Acids Symposium Series 1999, 41:95-98.

43. Rutherford K, Parkhill J, Crook J, Horsnell T, Rice P, Rajandream M-A, Barrell B: Artemis: sequence visualization and annotation. Bioinformatics 2000, 16:944-945

doi: $10.1186 / 1471-2180-10-137$

Cite this article as: Kirkup et al., Vibrio chromosomes share common history BMC Microbiology 2010, 10:137

\section{Submit your next manuscript to BioMed Central} and take full advantage of:

- Convenient online submission

- Thorough peer review

- No space constraints or color figure charges

- Immediate publication on acceptance

- Inclusion in PubMed, CAS, Scopus and Google Scholar

- Research which is freely available for redistribution

Submit your manuscript at www.biomedcentral.com/submit
C Biomed Central 